\title{
SIMPLE FORMULAS FOR THERMOELASTIC STRESSES IN TBC COATINGS
}

\author{
M. Ciavarella \\ CNR-IRIS \\ Bari, Italy \\ P. Decuzzi, V. L. Tagarielli, and G. P. Demelio \\ Dip Ing. Mecc e Gest. \\ Politecnico di Bari \\ Bari, Italy
}

\begin{abstract}
Thin coatings on metallic substrates are extensively used in mechanical, aerospace, microelectronic, optical, and medical applications. Coatings affect the surface properties of the system, while bulk properties and strength primarily depend on the substrate. In coated components subjected to thermal cycling, differences in physical properties and chemical nature between constituent parts of coating and substrate can cause thermal stresses, atomic diffusion, and chemical interactions, which decrease hightemperature stability and actual strength of the system. In this article, the microstructure of a thermal barrier coating is briefly described; then, the temperature field inside the coating is calculated from a transient or a steady periodic state analysis; hence, thermomechanical stresses are analytically determined for a double-layer system with planar or axisymmetric geometry. The present analysis leads to new formulas with which temperature and stress fields can be calculated.
\end{abstract}

Keywords TBC, thermoelastic stresses, thin films

Thin coatings are extensively used in many engineering applications. The main purpose of their use is to improve several surface properties of the coated component. Coatings can give both thermal and electrical insulation as well as protection from the environment; they can increase hardness and protect from wear and impact. In this article, attention will be paid to thermal barrier coatings, thin coatings used

Received 4 February 2002; accepted 11 November 2002.

M. Ciavarella is pleased to acknowledge CNR, Consiglio Nazionale delle Ricerche, for support of the network COMES (COmputational MEchanics of Solids) at CNR-IRIS. The authors acknowledge MIUR (Italian Ministry for Research) for support of the Excellence Centre in Computational Mechanics at Politecnico di Bari.

Address correspondence to Dr. M. Ciavarella, Primo Ricercatore, CNR-IRIS, Crocefisso 2B, Bari, 70126, Italy. 
mainly in hot parts of gas turbines to give thermal insulation. Thermal barrier coating (TBC) applications let us increase the temperature of the operating fluid and, therefore, the efficiency of gas turbines. In order to reach an appreciable temperature decrease, without modifying the external shape of components and their weight, coatings consist of very low thickness films, made of ceramic materials, which are deposited on metallic parts with physical or chemical techniques. They are characterized by both a very high fragility and a coefficient of thermal expansion (CTE) that is sensibly different from one of the metallic substrate. This structural nonhomogeneity and temperature gradients inside the components during their use cause thermomechanical stresses to appear. In the literature there are plenty of numerical and experimental approaches to this problem. Most analytical studies are based on crude approximations of the stress field [1,2]. In this article, thermal stresses are analytically determined; where the algebra was not too complicated, a transient analysis has been done, which has provided Eqs. (6), (7)-(10), and (12), which are expected to be useful in coated components design.

\section{STRUCTURE OF TBC COATINGS}

Looking at a section of a TBC coating (see [3]), we can distinguish several different layers (Figure 1): the base material (substrate), made of metallic alloys for high temperatures, the metallic bond coat (BC), and a ceramic top coat (TC); between the $\mathrm{BC}$ and the TC we can observe a very thin oxide layer (thermally grown oxides, TGO). The BC provides a physical and chemical link between ceramic coating and metallic substrate as well as protection from oxidation of substrate. Its chemical composition is commonly defined with the code MCrAlY, where $\mathrm{M}$ stands for one or more metals $(\mathrm{Ni}, \mathrm{Co}, \mathrm{Fe})$, among which the most common is nickel.

The TC is a ceramic layer (with a thickness of about $250-350 \mu \mathrm{m}$ ); its main function is to insulate the substrate. It is characterized by a very low thermal conductivity and stability at high temperatures. Materials used are alloys of oxides $\mathrm{ZrO}_{3}$ and $\mathrm{ZrO}_{2}$, plus a small amount $(6-7 \%)$ of yttrium oxide $\mathrm{Y}_{2} \mathrm{O}_{3}$. The CTE of this layer is considerably lower than the $\mathrm{CTE}$ of the $\mathrm{BC}$, and as a result thermal stresses are induced.

The TGO layer has a thickness between 5 and $50 \mu \mathrm{m}$, and it is generated by oxidation of BC aluminium. It provides good adhesion between TC and BC [4], but its growth during turbine operation, and the consequent volume expansion, causes residual stresses and reduces the fracture toughness of the coating $[5,6]$.

Table 1 shows the thermoelastic properties of TBC layers. It must be mentioned that $\mathrm{BC}$ has thermoelastic constants that are very similar to the properties of the metallic substrate. As a result, in a mathematical modeling of the coating, it can be neglected.

The main failure mode of $\mathrm{TBC}$ coatings is related to unstable crack propagation, which leads to delamination at the $\mathrm{BC}-\mathrm{TC}$ interface; the propagation is caused by the local stress field that arises during thermal cycling. The knowledge of the stress field is therefore very useful in predicting the lifetime of a coated component. 

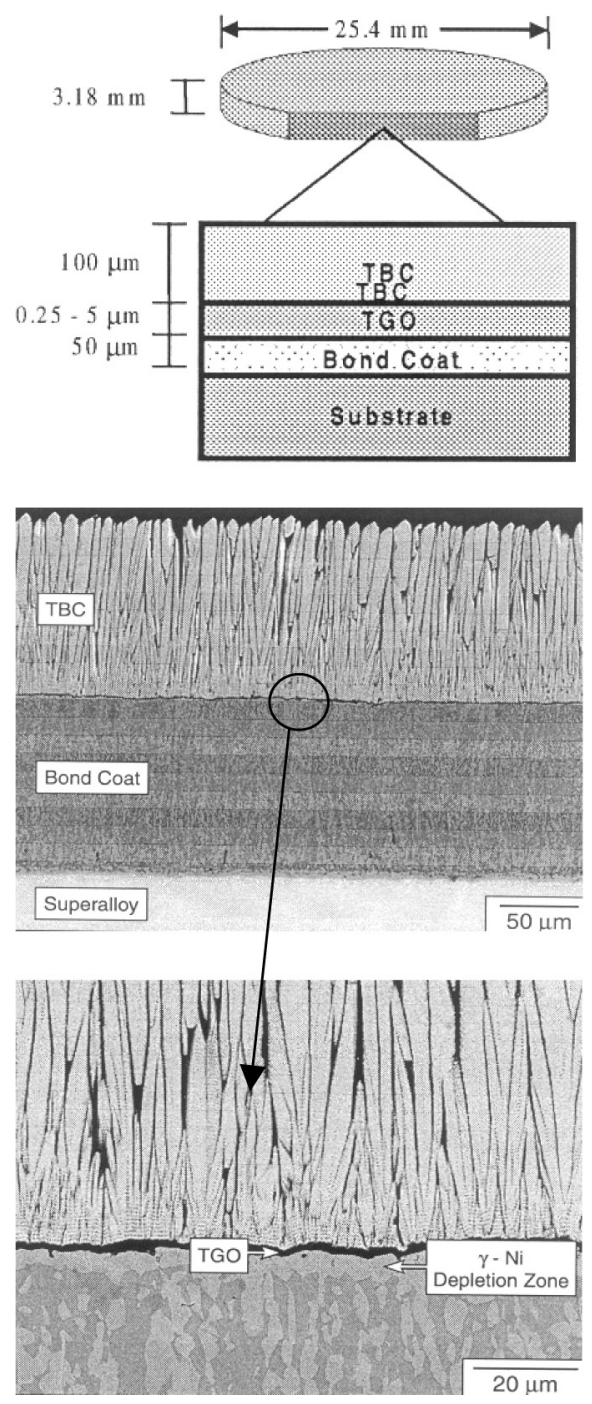

Figure 1. TBC structure [3].

\section{THERMO-ELASTIC STRESSES ANALYSIS}

Coated components undergo severe thermal cycling during their operation. Coating thickness is generally very low, and it can be neglected if compared with the thickness of the metallic substrate. If it is subjected to thermal cycling, dilatation tends to be different in the TC and the substrate; adhesion between TC and substrate often forces coating displacements to be zero, so that thermomechanical stresses arise. Therefore, coated components may be accurately modeled by a ceramic layer 
Table 1 Thermoelastic properties of TBC coatings

\begin{tabular}{lcll}
\hline & $\mathrm{E}[\mathrm{Mpa}]$ & $v$ & $\alpha\left[{ }^{\circ} \mathrm{C}^{-1}\right]$ \\
\hline BC & $200000-210000$ & $0.28-0.31$ & $1.45-1.55 \times 10^{-5}$ \\
TGO & $350000-360000$ & $0.26-0.28$ & $0.75-0.85 \times 10^{-5}$ \\
TC & $50000-60000$ & $0.10-0.15$ & $0.85-1.00 \times 10^{-5}$ \\
\hline
\end{tabular}

bonded to an elastic half-space. We will first consider the thermoelastic problem of a planar geometry; then we will extend our study to an axisymmetric geometry.

\section{Plane Geometry: Transient Analysis}

Consider an elastic half-space with a bonded ceramic layer (Figure 2). The temperature at which the body can be considered stressfree is $T_{0}$, and $T(x, t)$ is the temperature distribution inside the composite. Due to the symmetry of the problem we can write that

$$
\sigma_{i j}=0 \quad(i \neq j)
$$

The equilibrium equation ( $x$-axis) gives

$$
\sigma_{x x}=0
$$

By compatibility and constitutive equations, in which Eqs. (1) and (2) are taken into account, we can obtain equations that completely describe the strain and stress fields

$$
\begin{aligned}
\sigma_{y y}=\sigma_{z z} & =-\frac{E_{k} \alpha_{k}\left[T(x, t)-T_{0}\right]}{1-v_{k}} \\
e_{x x} & =\frac{1+v_{k}}{1-v_{k}} \alpha_{k}\left[T(x, t)-T_{0}\right] \quad k=1,2
\end{aligned}
$$

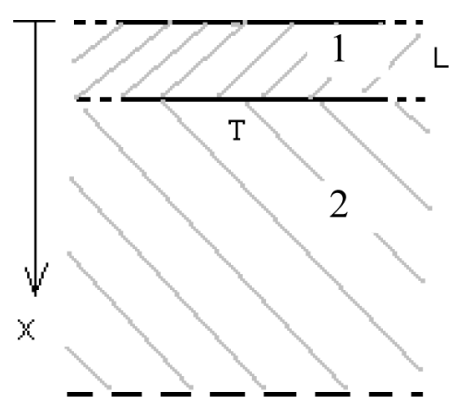

Figure 2. Ceramic layer bonded to an elastic half-space. 
where $k$ is 1 or 2 for the coating or the substrate, respectively. The temperature distribution must satisfy the heat conduction equation

$$
\nabla^{2} T(x, t)=\frac{1}{\kappa} \frac{\delta}{\delta t} T(x, t)
$$

(where $\kappa$ is the material's thermal diffusivity) with appropriate boundary and initial conditions, which in this case are

$$
\begin{aligned}
& T(x, t)=\bar{T}(t) \quad \text { for } \quad x=0 \\
& T(x, t)=T_{0} \quad \text { for } t=0
\end{aligned}
$$

where $\bar{T}(t)$ is the uniform temperature imposed on the external surface of the ceramic layer. Simple integration steps (see the appendix) give the temperature distribution inside the body

$$
\begin{aligned}
& T_{1}(x, t)-T_{0}=\int_{0}^{t}\left(\bar{T}(t-\lambda) \frac{\partial F_{1}}{\partial t}+\left(\frac{K_{1} h_{1}}{K_{2} h-K_{1} h_{2}} \bar{T}(t-\lambda)\right) \frac{\partial F_{2}}{\partial t}\right) d \lambda \quad \text { ceramic layer } \\
& T_{2}(x, t)-T_{0}=\int_{0}^{t}\left(\frac{K_{1} h_{1} g}{K_{2} h-K_{1} h_{2}} \bar{T}(t-\lambda)\right) d \lambda
\end{aligned}
$$

where $K_{1}$ and $K_{2}$ are thermal conductivities of coating and substrate, respectively, and $F_{1}, F_{2}, h, h_{1}, h_{2}$, and $g$ are known functions of $x$ and $(t-\lambda)$. Equations (6) and (3) give the stress and strain field inside the composite as a function of time.

\section{Plane Geometry: Steady Periodic State Analysis}

The integrals in Eqs. (6) can be very tedious. To obtain simpler formulas we consider a uniform but harmonic (with respect to time) temperature distribution and we restrict our attention after the transient period. Let $\bar{T}(t)$ be the temperature imposed on the free surface. We could also consider a generic periodic function $\bar{T}(t)$ as a sum of Fourier terms, say $T_{i} e^{j\left(\omega_{i} t+\varepsilon_{i}\right)}$ [7] and make use of the superposition principle. The analytic solution found is based on a formulation in Carslaw and Jaeger [1], it is found eventually that

$$
\begin{aligned}
\frac{T_{1}(x, t)}{T_{\text {sup }}}= & \frac{e^{j(\omega t)}}{1-e^{2\left(b_{1} l\right)(1+j)}}\left(e^{\left(b_{1} l\right) \frac{x}{l}(1+j)+\varepsilon_{1} j}-e^{\left(b_{1} l\right)\left(2-\frac{x}{l}\right)(1+j)+\varepsilon_{1} j}\right) \\
& +\frac{e^{j(\omega t)}}{1-e^{2\left(b_{1} l\right)(1+j)}} \frac{T_{\text {int }}}{T_{\text {sup }}}\left(e^{\left(b_{1} l\right)\left(1-\frac{x}{l}\right)(1+j)+\varepsilon_{2} j}-e^{\left(b_{1} l\right)\left(1+\frac{x}{l}\right)(1+j)+\varepsilon_{2} j}\right)
\end{aligned}
$$

for the coating and

$$
\frac{T_{2}(x, t)}{T_{\text {sup }}}=\frac{T_{\text {int }}}{T_{\text {sup }}} e^{-\left(b_{2} l\right)\left(\frac{x}{T}-1\right)} e^{j\left[(\omega t)+\varepsilon_{2}-\left(b_{2} l\right)\left(\frac{x}{T}-1\right)\right]}
$$


for the substrate, where

$$
b_{1}=\sqrt{\frac{\omega}{2 \kappa_{1}}} \quad b_{2}=\sqrt{\frac{\omega}{2 \kappa_{2}}}
$$

In Eq. (9), $\kappa_{1}$ and $\kappa_{2}$ are thermal diffusivities of coating and substrate, respectively, $T_{\text {sup }}$ is the temperature the amplitude on the composite's surface, and $\varepsilon_{1}$ is its phase angle. Constants $T_{\text {int }}$ and $\varepsilon_{2}$, which represent amplitude and phase angle of the temperature on the interface, are solutions of the nonlinear system of equations

$$
\begin{aligned}
& \varepsilon_{2}=\arctan \left(\frac{D}{C}\right)-\arctan \left(\frac{e^{2 b_{1} l} \sin \left(2 b_{1} l\right)}{e^{2 b_{1} l} \cos \left(2 b_{1} l\right)-1}\right)+\pi \\
& K_{2} b_{2} T_{\text {int }}=\frac{K_{1} b_{1} \sqrt{C^{2}+D^{2}}}{\sqrt{1-e^{4 b_{1} l}-2 \cos \left(2 b_{1} l\right)}} \\
& \text { with }\left\{\begin{array}{l}
C=2 T_{\text {sup }} e^{b_{1} l} \cos \left(b_{1} l+\varepsilon_{1}\right)-T_{\text {int }}\left(\cos \varepsilon_{2}+e^{2 b_{1} l} \cos \left(2 b_{1} l+\varepsilon_{2}\right)\right) \\
D=2 T_{\text {sup }} e^{b_{1} l} \sin \left(b_{1} l+\varepsilon_{1}\right)-T_{\text {int }}\left(\sin \varepsilon_{2}+e^{2 b_{1} l} \sin \left(2 b_{1} l+\varepsilon_{2}\right)\right)
\end{array}\right\}
\end{aligned}
$$

Dimensional analysis of Eqs. (10) gives

$$
\frac{T_{\text {int }}}{T_{\text {sup }}}=\frac{T_{\text {int }}}{T_{\text {sup }}}\left(b_{1} l, \frac{K_{1}}{K_{2}}, \frac{(\rho c)_{1}}{(\rho c)_{2}}\right) \quad \text { where } \frac{(\rho c)_{1}}{(\rho c)_{2}}=\frac{K_{1}}{K_{2}} \frac{\kappa_{2}}{\kappa_{1}}
$$

Equations (7)-(10) can be derived from Eq. (6) by assuming $\bar{T}(t)=T_{\text {sup }} e^{j\left(\omega t+\varepsilon_{1}\right)}$ and letting $t \rightarrow \infty$.

\section{Cylindrical Geometry: Steady-State Analysis}

A hollow metallic cylinder, coated by a ceramic cylindrical layer, has been analyzed (Figure 3). It has been assumed that the cylinders have infinite length so that all variables depend only on radius $r$. The elastic strain mismatch generates circumferential $\left(\sigma_{\theta \theta}\right)$, radial $\left(\sigma_{r r}\right)$, and axial $\left(\sigma_{z z}\right)$ stresses. As is shown in the appendix, and assuming $v_{1}=v_{2}=v$, it can be found that

$$
\begin{aligned}
\sigma_{\theta \theta}^{(2)}= & -\frac{E_{2}}{1-2 v} \Delta T \Delta \alpha \frac{\xi_{1}^{4} \chi\left(\xi_{2}^{2}-\xi_{1}^{2}\right)}{(1-\chi) \xi_{1}^{2}+\chi \xi_{2}^{2}-1} \\
\sigma_{\theta \theta}^{(1)}= & \frac{E_{2}}{1-2 v} \Delta T \Delta \alpha \frac{\xi_{1}^{4} \chi\left(\xi_{1}^{2}-1\right)}{(1-\chi) \xi_{1}^{2}+\chi \xi_{2}^{2}-1} \\
& \text { with } \xi_{1}=\frac{R_{2}}{R_{1}}, \xi_{2}=\frac{R_{3}}{R_{1}}, \chi=\frac{E_{1}}{E_{2}}
\end{aligned}
$$

The assumption $v_{1}=v_{2}=v$ provides a compact expression. It is generally not true in reality, but the influence of Poisson's modulus is very small. 


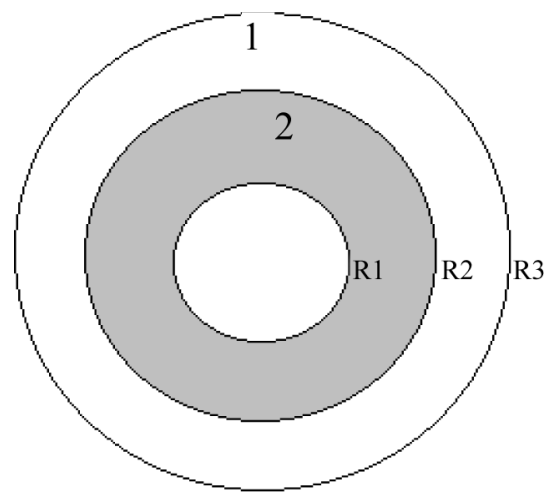

Figure 3. Cylindrical geometry.

\section{RESULTS AND DISCUSSION}

\section{Plane Geometry: Steady Periodic State Analysis}

The temperature distribution is calculated from Eqs. (7)-(10). Figure 4 shows a plot of temperature (nondimensional) as a function of time $(\omega t)$ and for several abscissas $(x / l)$; materials used are a zirconium oxide TC and a NiCrAlY BC $\left(\kappa_{1}=0.5 \mathrm{~m}^{2} / \mathrm{s}\right.$, $\kappa_{2}=1.9 \mathrm{~m}^{2} / \mathrm{s}$ ). The plot shows a progressive reduction of temperature amplitude inside the body and a delay of temperature fluctuation. Once the temperature field is completely known, Eqs. (3) provide stress and strain amplitudes and phases. Figure 5 shows a plot of inplane stress amplitude (nondimensional) with respect to $x / l$, where $\sigma_{\text {sup }}$ is the stress amplitude on the external surface. Notice that stress amplitude is a discontinuous function due to the nonhomogeneity of material properties. The

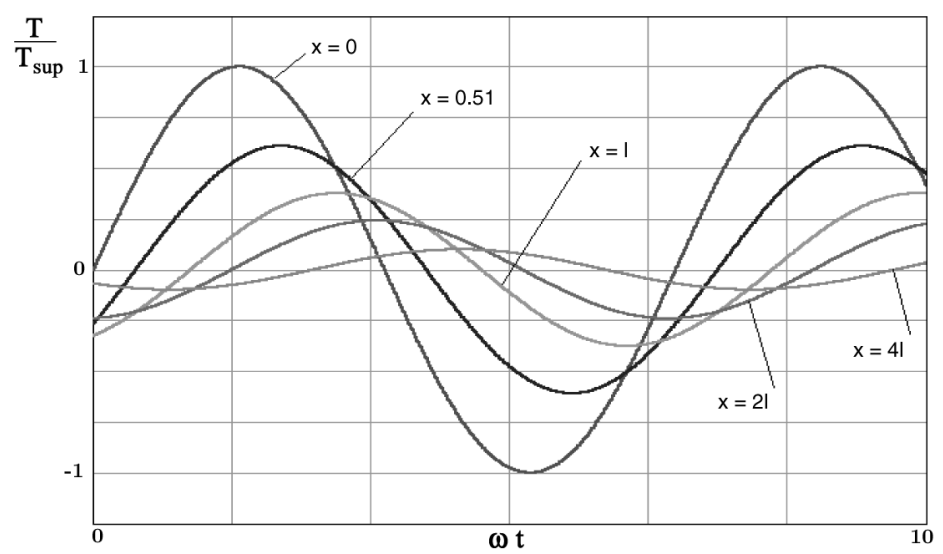

Figure 4. Temperature distribution as a function of time and nondimensional depth $(x / l)$. 

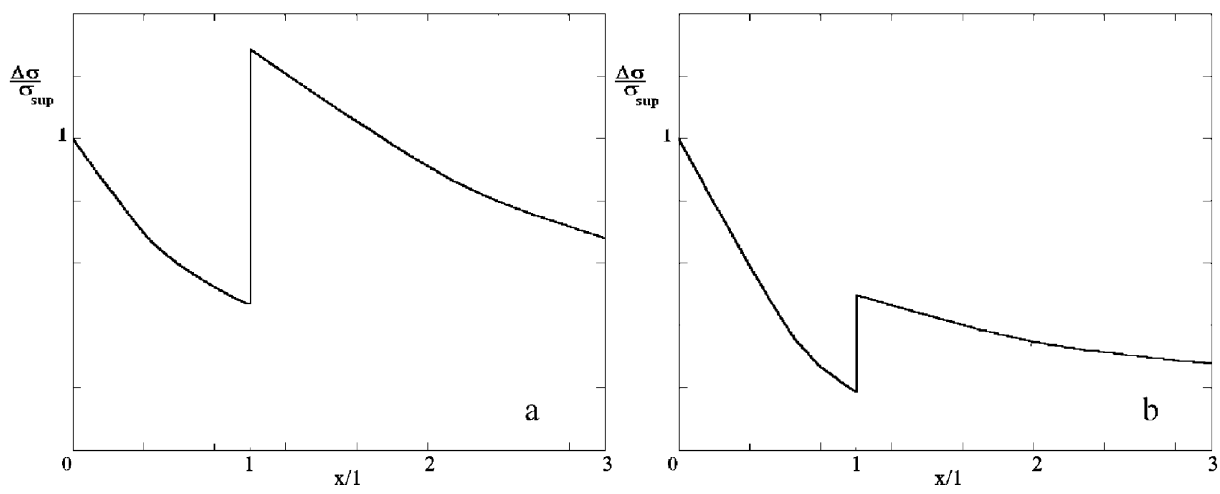

Figure 5. In-plane stress amplitude as function of depth $(x / l)$.

maximum value of transversal stresses is localized on the interface between BC and TC if the following condition is satisfied

$$
T_{\mathrm{int}}>T_{0}+\frac{E_{1} \alpha_{1}\left(1-v_{1}\right)}{E_{2} \alpha_{2}\left(1-v_{2}\right)}\left(T_{\text {sup }}-T_{0}\right)
$$

(as it usually happens) and its value is

$$
\sigma_{y y}^{(x=l)}=\sigma_{z z}^{(x=l)}=-\frac{E_{2} \alpha_{2}\left[T_{\mathrm{int}}-T_{0}\right]}{1-v_{k}}=-\frac{E_{2} \alpha_{2}}{1-v_{k}}\left[\left(\frac{T_{\text {int }}}{T_{\text {sup }}}\right) T_{\text {sup }}-T_{0}\right]
$$

If condition (13) is not satisfied, the maximum stress amplitude is localized on the external surface (Figure $5 b$ ). Equation (14) shows that the maximum stress amplitude is a function of $T_{\text {int }} / T_{\text {sup }}$ between the temperature amplitudes on the interface and on the surface. $T_{\text {int }} / T_{\text {sup }}$ is also an index of thermal insulation provided by ceramic coating, related to the parameter

$$
\Psi=\frac{T_{\text {sup }}-T_{\text {int }}}{T_{\text {sup }}}=1-\frac{T_{\text {int }}}{T_{\text {sup }}} \quad \Psi \in[0,1]
$$

For $\Psi=0$, the temperature amplitude on the external surface is equal to that on the interface and the thermal insulation is zero. On the other hand, for $\Psi=1$, the temperature amplitude on the interface is zero and the thermal insulation is maximum. Therefore, it is important to study variations of $T_{\text {int }} / T_{\text {sup }}$ when other parameters vary. Equation (11) shows that $T_{\text {int }} / T_{\text {sup }}$ is a function of $K_{1} / K_{2}, b_{1} l$, and $(\rho c)_{1} /(\rho c)_{2}$. In Figure $6 a$ it is shown that $T_{\text {int }} / T_{\text {sup }}$ rises if $K_{1} / K_{2}$ rises, is faster as $(\rho c)_{1} /(\rho c)_{2}$ is lower, and tends to one if $K_{1} / K_{2}$ tends to infinity. Similarly, $T_{\text {int }} / T_{\text {sup }}$ decreases if $(\rho c)_{1} /(\rho c)_{2}$ rises and is faster as $K_{1} / K_{2}$ is lower (Figure 6b). Finally, a plot of $T_{\text {int }} / T_{\text {sup }}$ as a function of $b_{1} l$ is presented in Figure 7 . If $K_{1} / K_{2}$ decreases and $(\rho c)_{1} /(\rho c)_{2}$ and $b_{1} l$ rise, stress amplitude decreases and thermal insulation rises. In particular, for typical values of governing parameters for TBC $\left(K_{1} / K_{2} \cong 0.25\right.$; $\left.(\rho c)_{1} /(\rho c)_{2} \cong 1.4 ; b_{1} l \cong 0.1\right)$, it can be found that $T_{\text {int }} / T_{\text {sup }}$ decreases by $50 \%$ if 


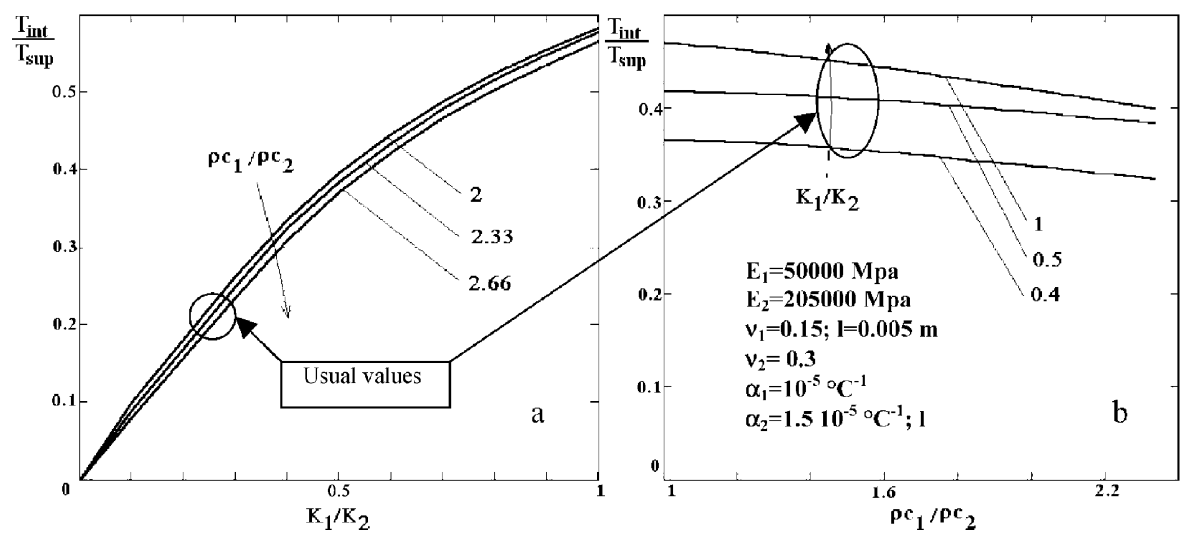

Figure 6. $T_{\text {int }} / T_{\text {sup }}$ as a function of governing parameters.

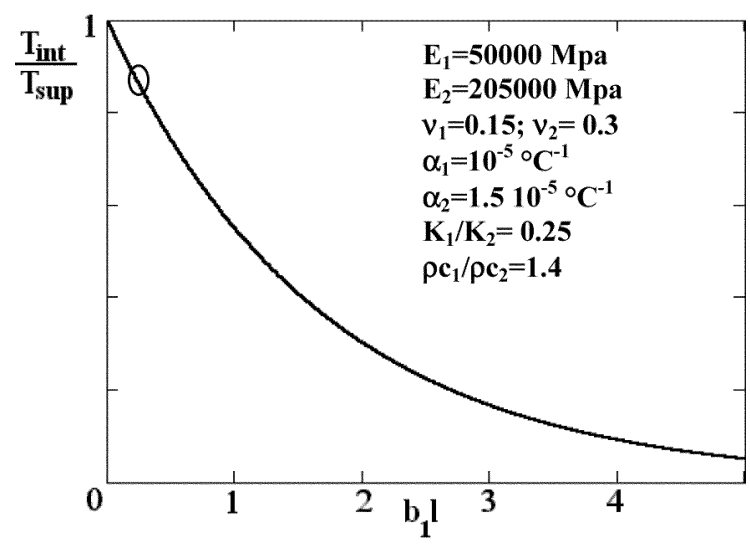

Figure 7. $T_{\mathrm{int}} / T_{\text {sup }}$ as a function of $b_{1} l$.

$K_{1} / K_{2}$ goes down by $50 \%$, while the same ratio goes down by 3 to $4 \%$ if $(\rho c)_{1} /(\rho c)_{2}$ rises by $50 \%$. Furthermore, appreciable variations of $T_{\text {int }} / T_{\text {sup }}$ can be obtained by modifying coating thickness $l$, which is restricted by the deposition techniques used and thermal diffusivity, which appears in $b_{1}$.

\section{Cylindrical Geometry: Steady-State Analysis}

Equations (12) give circumferential stresses in a cylindrical bilayer. Stresses are proportional to $\Delta T \Delta \alpha$ and have opposite sign in the coating and in the substrate, tending to zero when $\chi$ tends to zero. Figure 8 shows nondimensional circumferential stresses $\left(\beta_{i}=\sigma_{\theta \theta(i)}(1-2 v) /\left(E_{2} \Delta T \Delta \alpha\right)\right)$ as functions of coating thickness. In the substrate, their modulus rises if $t_{1}$ rises and is as higher as $\chi$ is big; $\sigma_{\theta \theta}$ in the ceramic layer tends to zero if $t_{1}$ tends to infinity; on the other hand, if $t_{1}$ is very small, $\sigma_{\theta \theta}$ stresses in the TC blow up. 

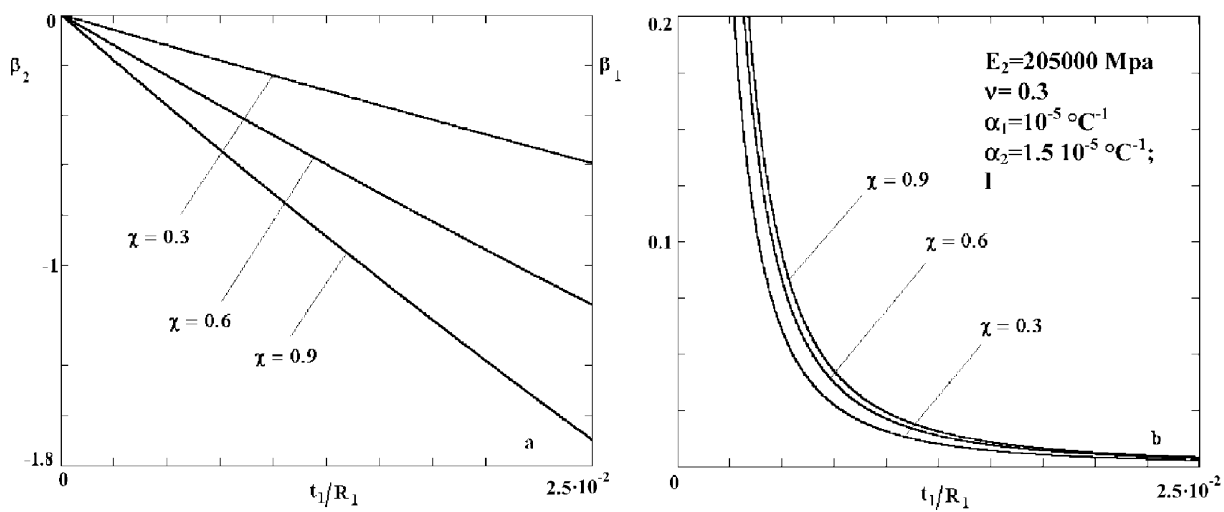

Figure 8. $\beta_{2}$ and $\beta_{1}$ as functions of the $t_{1} / R_{1}$ ratio and of the nondimensional parameter $\chi$.

\section{CONCLUSIONS}

After a brief introduction about TBC coatings and their applications and problems, an analytical investigation of thermomechanical stresses in coated components has been done. Thermoelastic problems for both planar and cylindrical TBC geometries have been solved, giving simple formulae to calculate temperature, stress, and strain distributions. Some of Eqs. (6), (7)-(10), and (12) were not present in the literature previously. Starting from the aforementioned equations, the functional dependence of the stress and temperature distributions has been found. For plane TBCs with harmonic temperature imposed on the external surface, it has been shown that by increasing thermal insulation, the stress field becomes less severe and the coating toughness increases. Coatings should have low thermal conductivity or big calorific capacity in order to reduce $K_{1} / K_{2}$ or to raise $(\rho c)_{1} /(\rho c)_{2}$ : decreasing $K_{1} / K_{2}$ or increasing $(\rho c)_{1} /(\rho c)_{2}$ by $50 \%, T_{\text {int }} / T_{\text {sup }}$ will reduce by $50 \%$ or 3 to $4 \%$, respectively. Finally, for a cylindrical TBC, the steady-state problem has been solved, presenting formulae which give circumferential stresses as functions of coating thickness and material properties. It has also been found that the stress value in the substrate increases with coating thickness and becomes higher as the value of the ratio $\chi$ increases.

\section{REFERENCES}

1. H. S. Carslaw, and J. C. Jaeger, Conduction of Heat in Solids, Clarendon Press, Oxford, 1947.

2. B. A. Boley, and J. H. Weiner, Theory of Thermal Stresses, John Wiley \& Sons, New York, 1990.

3. J. W. Hutchinson, A. G. Evans, and S. R. Choi, Delamination of Multilayer Thermal Barrier Coatings, Mech. Mat., vol. 31, pp. 431-447, 1999.

4. Y. C. Tsui, and T. W. Clyne, Adhesion of Thermal Barrier Coating Systems and Incorporation of an Oxidation Barrier Layer, Proc. 9th Nat. Thermal Spray Conference, pp. 275-284, 1996.

5. R. A. Handoko, J. L. Beuth, G. H. Meier, F. S. Pettit, and M. J. Stiger, Mechanism for Interfacial Toughness Loss in Thermal Barrier Coatings Systems, Proc. Mat. Div. Symposium on Durable Surfaces, 2000 . 
6. S. Q. Nusier, G. M. Newaz, and Z. A. Chaudhury, Experimental and Analytical Evaluation of Damage Processes in Thermal Barrier Coatings, Int. J. Solids Structures, vol. 37, pp. 2495-2506, 1998.

7. I. N. Sneddon, Fourier Transforms, Dover Publications, New York, 1995.

8. J. R. Barber, Elasticity, Kluwer Academic Publishers, Dordrecht, 1996.

\section{APPENDIX A}

\section{Plane Geometry: Transient Analysis}

In the literature [1], solutions exist for the transient temperature field (i) for a layer that is initially at $T=0$ everywhere and with given generic temperature functions on its faces and (ii) for an elastic half-space with $T=0$ initially and with a prescribed temperature on the external surface. According to the coordinate systems presented in Figure A1, they can be rewritten as

$$
\begin{aligned}
& T_{1}\left(x_{1}, t\right)=\int_{0}^{t}\left(\phi_{1}(\lambda) \frac{\partial}{\partial t} F_{1}\left(x_{1}, t, \lambda\right)+\phi_{2}(\lambda) \frac{\partial}{\partial t} F_{2}\left(x_{1}, t, \lambda\right)\right) d \lambda \\
& T_{2}\left(x_{2}, t\right)=\int_{0}^{t} \phi_{2}(t, \lambda) \frac{\partial}{\partial t} F\left(x_{2}, t, \lambda\right) d \lambda
\end{aligned}
$$

where

$$
\begin{aligned}
F\left(x_{2}, t, \lambda\right) & =\frac{2}{\sqrt{\pi}} \int_{\frac{x_{2}}{2 \sqrt{\kappa_{2}(t-\lambda)}}}^{\infty} e^{-\xi^{2}} d \xi \\
F_{1}\left(x_{1}, t, \lambda\right) & =1-\frac{x}{l}-\frac{2}{\pi} \sum_{n=1}^{\infty}\left(e^{\left(\frac{-\kappa_{1} n^{2} \pi^{2}(t-\lambda)}{l^{2}}\right)} \frac{1}{n} \sin \left(\frac{n \pi x}{l}\right)\right) \\
F_{2}\left(x_{1}, t, \lambda\right) & =\frac{x}{l}+\frac{2}{\pi} \sum_{n=1}^{\infty}\left(\cos (n \pi) e^{\left(\frac{-\kappa_{1} n^{2} \pi^{2}(t-\lambda)}{l^{2}}\right)} \frac{1}{n} \sin \left(\frac{n \pi x}{l}\right)\right)
\end{aligned}
$$

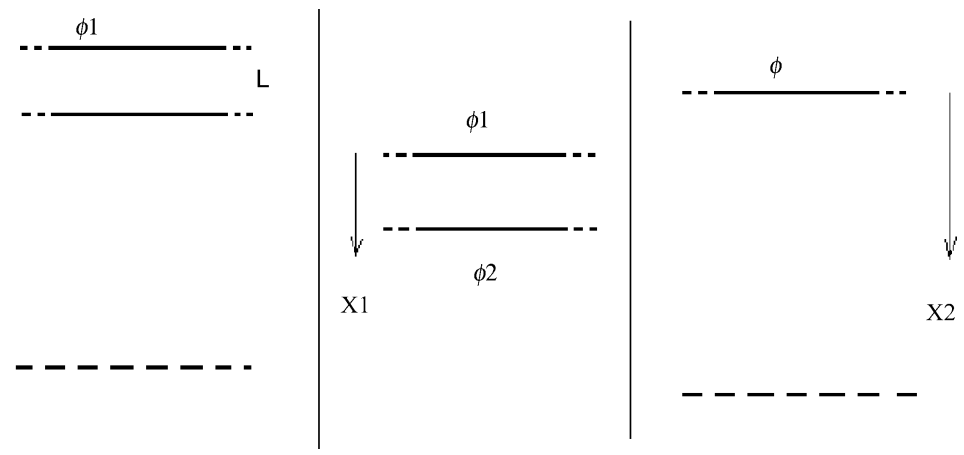

Figure A1. Used coordinate systems. 
In the last set of equations, continuity of temperature at the interface $\left(\phi_{2}=\phi\right)$ has already been imposed. We then define the functions

$$
r_{i}=\frac{\partial}{\partial t} F_{i} \quad g_{i}=\frac{\partial}{\partial x_{1}} F_{i} \quad h_{i}=\frac{\partial}{\partial t} g_{i} \quad g=\frac{\partial}{\partial t} F \quad h=\frac{\partial}{\partial x_{2}} g
$$

A further condition to be respected by temperature distributions is that thermal flux has to be continuous at the interface between coating and substrate. If the generic thermal flux is

$$
q_{i}\left(x_{i}, t\right)=-K_{i} \frac{\partial T_{i}}{\partial x_{i}}
$$

we can write

$$
\begin{aligned}
q_{1}(l, t) & =q_{2}(0, t) \Rightarrow-K_{1} \int_{0}^{t}\left(\phi_{1} h_{1}(l, t, \lambda)+\phi_{2} h_{2}(l, t, \lambda)\right) d \lambda=-K_{2} \int_{0}^{t} \phi_{2} h(0, t, \lambda) d \lambda \\
& \Rightarrow \phi_{2}(t, \lambda)=\frac{K_{1} h_{1}(l, t, \lambda)}{K_{2} h(0, t, \lambda)-K_{1} h_{2}(l, t, \lambda)} \phi_{1}(\lambda)
\end{aligned}
$$

Equation (A.5) gives the temperature at the interface as a function of the imposed boundary temperature, of the material properties, and of the ceramic layer thickness l. By substituting this expression into Eqs. (A.1), after simple analytical steps, Eq. (6) is obtained.

\section{Plane Geometry: Steady Periodic State Analysis}

After the transient period, temperature inside the body will have the same frequency of temperature imposed on the external surface and an amplitude that will depend on the depth $(x / l)$. Its analytical expression will be

$$
T(x, t)=y(x) e^{j(\omega t+\phi)}
$$

By substituting the last expression in the heat conduction equation, we have

$$
\begin{aligned}
\frac{\partial^{2} y}{\partial x^{2}}-\frac{\omega j}{\kappa} y & =0 \Rightarrow y(x)=c_{1} e^{b x(1+j)}+c_{2} e^{-b x(1+j)} \\
& \Rightarrow T(x, t)=\left(c_{1} e^{b x(1+j)}+c_{2} e^{-b x(1+j)}\right) e^{j(\omega t+\phi)} \quad \text { where } b=\sqrt{\frac{\omega}{2 \kappa}}
\end{aligned}
$$

From this general solution, we can obtain the temperature distribution for a halfspace with an imposed temperature on the external surface $\left(T_{\text {int }} e^{j\left(\omega t+\varepsilon_{2}\right)}\right.$ at $\left.x_{2}=0\right)$ and for a layer with imposed temperature distributions on its two faces $\left(T_{\text {sup }} e^{j\left(\omega t+\varepsilon_{1}\right)}\right.$ at $x_{1}=0, T e^{j\left(\omega t+\varepsilon_{2}\right)}$ at $\left.x_{1}=l\right)$. The aforementioned expressions are 


$$
\begin{aligned}
T\left(x_{2}, t\right)= & T_{\mathrm{int}} e^{-b_{2} x_{2}} e^{j\left(\omega t+\varepsilon_{2}-b_{2} x_{2}\right)} \\
T\left(x_{1}, t\right)= & \frac{e^{j \omega t}}{1-e^{2 b_{1} l(1+j)}} T_{\sup }\left(e^{b_{1} x_{1}(1+j)+\varepsilon_{1} j}-e^{\left(2 b_{1} l-b_{1} x_{1}\right)(1+j)+\varepsilon_{1} j}\right) \\
& +\frac{e^{j \omega t}}{1-e^{2 b_{1} l(1+j)}} T\left(e^{b_{1}\left(l-x_{1}\right)(1+j)+\varepsilon_{2} j}-e^{b_{1}\left(l+x_{1}\right)(1+j)+\varepsilon_{2} j}\right)
\end{aligned}
$$

where the suffix 1 is for the layer and 2 is for the half-space. Continuity of temperature on the interface gives $T=T_{\text {int }}$. A further boundary condition for Eqs. (A.8) is that thermal flux has to be continuous on the interface

$$
q_{1}(l, t)=q_{2}(0, t)
$$

This is an equation between two rotating vectors; by equating their amplitude and phase, we obtain relations (10).

\section{Cylindrical Geometry: Steady-State Analysis}

Consider a hollow circular disk with an infinite axial length, and impose a uniform temperature variation $\Delta T$ from a reference temperature $T_{0}$; constitutive and compatibility equations give

$$
\begin{aligned}
\sigma_{r r} & =E_{1}\left(\frac{\partial u_{r}}{\partial r}-\alpha \Delta T\right)+E_{2} \frac{u_{r}}{r}-E_{3} \alpha \Delta T \\
\sigma_{\theta \theta} & =E_{1}\left(\frac{u_{r}}{r}-\alpha \Delta T\right)+E_{2} \frac{\partial u_{r}}{\partial r}-E_{3} \alpha \Delta T \\
\sigma_{z z} & =E_{2}\left(\frac{\partial u_{r}}{\partial r}+\frac{u_{r}}{r}\right)+E_{4} \alpha \Delta T
\end{aligned}
$$

where

$$
E_{1}=\frac{(1-v) E}{(1+v)(1-2 v)} \quad E_{2}=\frac{v E}{(1+v)(1-2 v)} \quad E_{3}=2 E_{2} \quad E_{4}=\frac{E}{2 v-1}
$$

By substituting (A.10) into the equilibrium equation (A.12), we write

$$
\begin{gathered}
\sigma_{\theta \theta}-\sigma_{r r}=r \frac{\partial \sigma_{r}}{\partial r} \quad \frac{\partial \sigma_{\theta \theta}}{\partial \theta}=0 \quad \frac{\partial \sigma_{z z}}{\partial z}=0 \\
\frac{\partial^{2} u_{r}}{\partial r^{2}}+\frac{1}{r} \frac{\partial u_{r}}{\partial r}-\frac{u_{r}}{r^{2}}=0
\end{gathered}
$$

Relation (A.13) is an Euler equation, and it has the general solution

$$
u_{r}(r)=\frac{c_{1}}{r}+c_{2} r
$$


If boundary conditions are a uniform traction at external and internal radii $\left(\sigma_{1}, \sigma_{2}\right)$, integration constants can be calculated:

$$
c_{1}=\frac{(1+v) R_{1}^{2} R_{2}^{2}\left(\sigma_{1}-\sigma_{2}\right)}{E\left(R_{1}^{2}-R_{2}^{2}\right)} \quad c_{2}=\frac{(1+v)(1-2 v)\left(R_{1}^{2} \sigma_{1}-R_{2}^{2} \sigma_{2}\right)}{E\left(R_{1}^{2}-R_{2}^{2}\right)}+\alpha(1+v) \Delta T
$$

$c_{1}$ is usually one thousand times lower than $c_{2}$, so it can be neglected (provided that $R_{1}$ and $R_{2}$ values are not very similar). If we have two hollow circular cylinders bonded to each other, as shown in Figure 3, the last relations can be used to find integration constants $c_{1}$ and $c_{2}$ for both the substrate and ceramic layers. Let us use suffixes (2) and (1) for substrate and layer, respectively, and assume that surfaces $r=R_{1}$ and $r=R_{3}$ are stressfree. We impose

$$
u_{(2)}\left(R_{2}\right)=c_{2(2)} R_{2}=u_{1}\left(R_{2}\right)=c_{2(1)} R_{2}
$$

Furthermore, radial stresses at the interface must be continuous functions, so we have to write

$$
\sigma_{1(2)}=\sigma_{2(1)}=0 \quad \sigma_{2(2)}=\sigma_{1(1)}=\sigma_{\mathrm{int}}
$$

Equations (A.16) and (A.17) yield the radial stress at the interface, which is needed to evaluate the integration constants $c_{2(2)}, c_{2(1)}$. Once these constants are known, the displacement field is also completely determined (A.14); by substituting its expression in Eqs. (A.10), we eventually find relations (12). 